\title{
Primary Melanoma of the Right Colon Presenting With Gastrointestinal Bleeding
}

\author{
Busuttil Gerald ${ }^{1}{ }^{1 *}$, Blazic $\operatorname{Ivan}^{1}$, Caruana Dingli Gordon ${ }^{1}$, Agius John ${ }^{1}$ and Babic Darko ${ }^{2}$ \\ ${ }^{1}$ Department of Surgery, Mater Dei Hospital, Tal-Qroqq, MSD 2090, Malta \\ ${ }^{2}$ Department of Pathology, Mater Dei Hospital Tal-Qroqq, MSD 2090, Malta
}

\begin{abstract}
A 73 year old female presented with painless fresh rectal bleeding and anaemia. At colonoscopy, a large mass arising from the right colon was visualised and biopsied. Histological evaluation established a diagnosis of melanoma. Thorough clinical examination confirmed the presence of primary localised melanoma which was amenable to resection.

At laparotomy, right hemicolectomy and lymphadenectomy were performed. Two years after her surgery, our patient is well without any evidence of disease recurrence.

Primary melanoma of the colon is a very rare clinical entity and limited data are available regarding its pathogenesis, treatment and outcome. Primary surgical resection in localised disease appears to be the mainstay of treatment, although the role of adjuvant therapy is still to be established.
\end{abstract}

Keywords: Melanoma, Right colon, Gastrointestinal bleeding, Anaemia, Colonoscopy.

\section{BACKGROUND}

In most documented cases of melanoma of the gastrointestinal tract, the alimentary tract has been involved secondarily from a cutaneous or ocular malignancy. Primary melanoma of the colon is a rare entity, with very few cases reported in the medical literature. This case report illustrates once such case of primary melanoma of the right colon presenting at our institution.

\section{CASE PRESENTATION}

A 73 year old female was referred to the surgical outpatient's clinic after two episodes of painless fresh bleeding per rectum. This bleeding was not accompanied by change in bowel habit or systemic features of malignancy. Physical examination was unremarkable. The patient had no relevant family history.

Baseline blood investigations revealed a hypochromic microcytic anaemia. This finding prompted further investigation, with tumour markers and oesophagogastroduodenoscopy being normal.

Colonoscopy was the next diagnostic step in accordance with the British Society of Gastroenterology Guidelines for the management of iron deficiency anaemia [1]. This showed a large pedunculated and pigmented necrotic mass arising

*Address correspondence to this author at the Department of Surgery, Mater Dei Hospital, Tal-Qroqq, MSD 2090, Malta; Tel: +356 99228379;

Fax:+356 21240176; E-mail: gerald.busuttil@gov.mt from the right colon. Biopsy showed malignancy with an immunohistochemical profile favouring melanoma. CT scan of the thorax and abdomen (Figs. 1 and 2) confirmed the presence of a large tumourous mass in the right colon without distant spread or lymph node metastasis.

The past medical history was reviewed, and given that our patient never had suspicious cutaneous lesions excised, she underwent through dermatological, ENT, gynaecological and ophthalmic assessments to exclude primary tumour elsewhere.

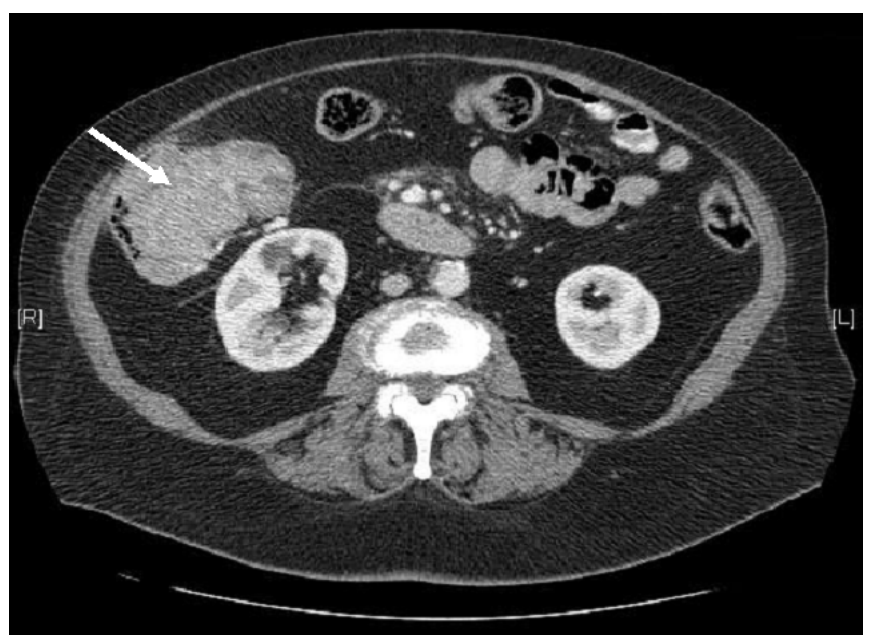

Fig. (1). Staging CT scan showing large heterogeneous mass of the right colon with no evidence of local invasion. 


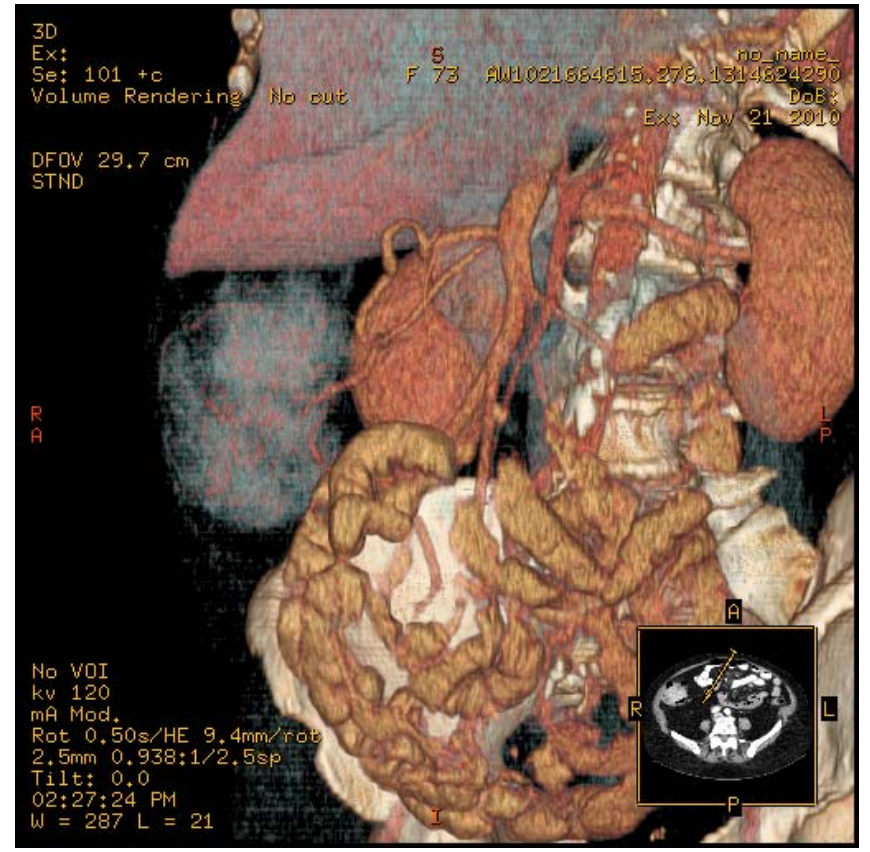

Fig. (2). 3D CT reconstruction showing vascular solid tumour at the hepatic flexure with feeding artery from superior mesenteric artery. At laparotomy the imaging findings were confirmed, with a large non-obstructive solid mass at the hepatic flexure with no palpably enlarged regional lymph nodes.

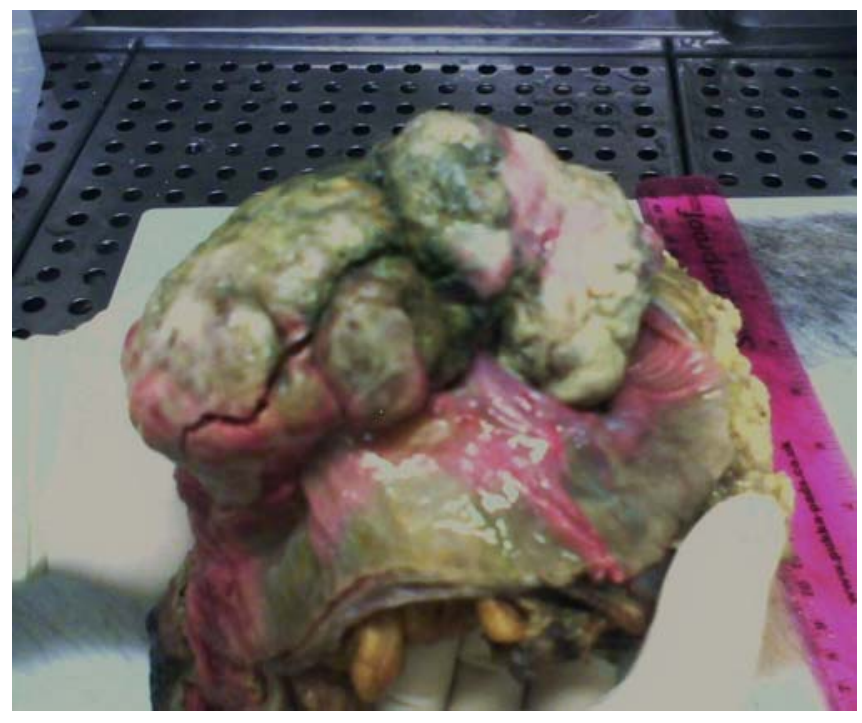

Fig. (3). Operative specimen: right colon with large intraluminal pedunculated and pigmented mass.

After discussion at a multidisciplinary meeting, an extended right hemicolectomy and lymphadenectomy were performed (Fig. 3). At laparotomy there were no liver, omental or peritoneal deposits and no regional lymph nodes were macroscopically involved by the neoplastic process.

Histopathological examination of the resection specimen confirmed the previous biopsy findings, with a $110 \mathrm{~mm}$ exophytic, ulcerated and pigmented lesion of the right colon exhibiting characteristic melanosis suggestive of melanoma (Fig. 4). The tumour was noted to invade the muscularis pro-

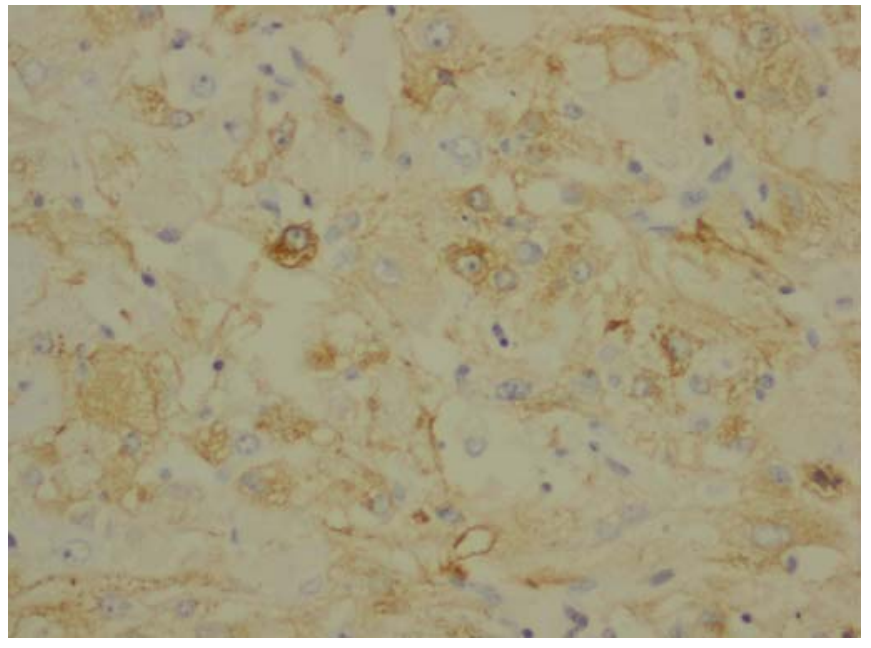

Fig. (4). Histological slide showing malignant cells exhibiting positive Melan A (A11), HMB45 (A4, A11) and S100 staining typical of malignant melanoma (20x).

pria, with two of the eighteen resected lymph nodes showing microscopic spread from the primary tumour (pathological stage T2 N1b M0, National Comprehensive Cancer Network ${ }^{\circledR}$ prognostic group IIIA).

The patient was followed up according to the National Comprehensive Cancer Network guidelines for follow-up of colorectal cancer [2]. After discussion with the medical oncologist it was decided not to give this patient further adjuvant therapy.

This is the first and only case of primary melanoma of the bowel presenting and treated at our institution and two years after surgery there is no evidence of local recurrence or distant metastasis on follow up imaging and endoscopy.

\section{DISCUSSION}

Primary melanoma of the gastrointestinal tract is a rare tumour; involvement of the alimentary tract is usually secondary to metastatic spread from primary tumours [3]. In an autopsy study Avital et al showed that up to $60 \%$ of patients with melanoma have gastrointestinal involvement [4].

Reintgen et al dispute the existence of primary melanoma, arguing that these cases represent distant metastasis from an occult primary, given that melanoma is the most common malignancy having the potential to metastasize to the gastrointestinal tract [5].

Because of this controversy, Sachs et al. proposed four criteria that characterise primary, rather than metastatic, melanoma; primary intestinal melanoma: 1) is a solitary lesion, 2) has no metastatic lesion to other organs, 3) has precursor lesions or melanosis histologically, and 4) has a disease-free survival period of at least 12 months after diagnosis [6]. Our patient fulfils all of the criteria described by Sachs.

Most cases of primary melanoma of the gastrointestinal tract occur in the oesophagus and anorectum, probably because melanocytes can be found in these regions [7]. Because the small and large intestines do not normally contain 
melanoblasts, the pathogenesis of primary colonic melanoma is a controversial issue [8].

Neural crest cells are however found extensively in the colon, and in vitro studies have shown that these cells have the potential to differentiate into mature melanocytes [9] by a process of ectodermal differentiation and migration during embryological development [10].

The occurrence of primary melanoma of the colon is relatively rare, with 12 cases reported in the literature to date, [11] with the right colon and the caecum being the commonest sites. This contrasts with metastatic melanoma, which seems to preferentially involve the ascending and descending colon [12].

From a clinical perspective abdominal pain (58\%) and weight loss (50\%) are the most common modes of presentation. Gastrointestinal bleeding and bowel obstruction are also relatively common at presentation [12].

Due to the rarity of such lesions no clinical practice guidelines exist on their management. Aggressive surgical intervention with wide excision margins is the first line treatment for localised resectable disease [11]. The role of adjuvant or neoadjuvant therapy in the setting of primary melanoma is also debatable as melanoma is generally chemoresistant. Work by Tessier et al demonstrated no statistically significant benefit in patients who received postoperative adjuvant chemotherapy [12].

Data on survival following colectomy for primary tumours is sparse and the true effect of surgery is unknown. Most published series refer to metastatic melanoma of the bowel, with patients having positive nodes faring worse than their node-negative counterparts. ${ }^{[12]}$ In the only series of patients who had surgery for primary melanoma in various sites of the colon, the average time to recurrence was 2.59 years. ${ }^{[11]}$ Presentation with obstruction or perforation was associated with a worse prognosis.

\section{CONCLUSION}

The scarcity of data regarding the natural history and tumour biology of primary colonic melanoma makes treatment and follow up empirical rather than evidence-based. Given the low incidence of this condition, it is improbable that large randomised studies on the matter will be feasible. Referral to tertiary centres may allow the setting up of clinical trials which hopefully would shed some light on the matter.

\section{CONFLICT OF INTEREST}

The author(s) confirm that this article content has no conflicts of interest.

\section{ACKNOWLEDGEMENT}

None declared.

\section{REFERENCES}

[1] Goddard AF, James MW, McIntyre AS, Scott B. British Society of Gastroenterology Guidelines for the management of iron deficiency anaemia - Gut 2011; 60:1309-16.

[2] NCCN. Clinical Practice Guidelines in Oncology - Colon Cancer Version 3. - NCCN.org 2012.

[3] Frost DB, Mercado PD, Tyrell JS. Small bowel cancer. A 30- year review. Ann Surg Oncol 1994; 1: 290-5.

[4] Avital S, Romaguera RL, Sands L, Marchetti F, Hellinger MD. Primary malignant melanoma of the right colon. Am Surg 2004; 70: 649-51.

[5] Reintgen DS, Thompson W, Garbutt J, Siegler HF. Radiologic, endoscopic, and surgical considerations of melanoma metastatic to the gastrointestinal tract. Surgery 1984; 95: 635-9.

[6] Sachs DL, Lowe L, Chang AE, et al. Do primary small intestinal melanomas exist? Report of a case. J Am Acad Dermatol 1999; 41: 1042-4.

[7] Serin G, Doganavsargil B, Caliskan C, Akalin T, Sezak M, Tunyurek M. Colonic malignant melanoma, primary or metastatic? Case report. Turkish J Gastroenterol 2010; 21: 45-9.

[8] Delcore R, Friesen SR. Embryologic concepts in the APUD system. Semin Surg Oncol. 1993; 9 (5): 349-361.

[9] Jacobs-Cohen RJ, Wade PR, Gershon MD. Suppression of the melanogenic potential of migrating neural crest-derived cells by the branchial arches. Anat Rec 2002; 268:16-26.

[10] Mori D, Satoh T, Nakafusa Y, Tanaka M, Miyazaki K, Tokunaga O. Primary colonic malignant melanoma. Pathol Int 2006; 56:7448.

[11] Khalid U, Saleem T, Mallick Imam A , Rizwan Khan M. Pathogenesis, diagnosis and management of primary melanoma of the colon. World J Surg Oncol 2011, 9:14.

[12] Tessier DJ, McConnell EJ, Young-Fadok T, Wolff BG. Melanoma metastatic to the colon: case series and review of the literature with outcome analysis. Dis Colon Rectum 2003; 46: 41-7. 